\title{
Change in vitamin B12 level in breast milk according to the week of delivery in Turkish women
}

\author{
Fatih Yesildal', (ㄷ) Esin Koc², Hakan Ozturk², () Taner Ozgurtas ${ }^{3}$ \\ 'Department of Medical Biochemistry, Istanbul Medeniyet University Goztepe Teaching and Research Hospital, Istanbul, Turkey \\ ${ }^{2}$ Department of Pediatrics, Gazi University Faculty of Medicine, Ankara, Turkey \\ ${ }^{3}$ Department of Medical Biochemistry, University of Health Sciences, Gulhane Faculty of Medicine, Ankara, Turkey
}

\begin{abstract}
Objectives: Vitamin B12 is one of the most important micronutrients in breast milk, as a deficiency can lead to growth and developmental retardation in infants. The aim of this study was to investigate differences in the level of vitamin B12 in breast milk in preterm, late preterm, and term groups according to the number of gestational weeks at delivery, and how vitamin B12 content in breast milk changed during the first 28 days of lactation.

Methods: A total of 30 mothers ( $n=10$ each for preterm, late preterm, and term delivery groups) were included in the study. Breast milk samples were collected from the participants on day 3, 7, and 28 after delivery. The level of vitamin B12 was analyzed in each sample using the Advia Centaur XP autoanalyzer (Siemens Healthineers GmbH, Erlangen, Germany). Analysis of variance (ANOVA) and post hoc Tukey tests were used for group comparisons.

Results: There was a significant difference in the level of vitamin B12 between the 3 groups based on the week of delivery (ANOVA test, $p=0.012$ ). In addition, regardless of the number of days of lactation, a significant difference was found between the preterm $(954.1 \pm 462.8 \mathrm{ng} / \mathrm{L})$ and term $(647 \pm 253.2 \mathrm{ng} / \mathrm{L})$ groups in the post hoc Tukey pairwise comparison results $(p=0.010)$. The vitamin $B 12$ level in the $28^{\text {th }}$ day breast milk samples was significantly different between the preterm group $(1104.1 \pm 431.9 \mathrm{ng} / \mathrm{L})$ and the term group $(547.1 \pm 274.4 \mathrm{ng} / \mathrm{L})(\mathrm{p}=0.030)$.

Conclusion: In the term delivery group, the highest level of vitamin B12 was seen in the colostrum, and was lower in transition milk and mature milk. In contrast, in the preterm delivery group, mature milk contained a higher level of vitamin B12 than the colostrum. The vitamin B12 level in breast milk in the preterm group was higher on the $28^{\text {th }}$ day of lactation compared with the term group. This may be a compensation mechanism to prevent possible retardation of growth in the preterm group. According to these results, preterm delivery infants do not require additional vitamin B12 supplementation unless there is a pathology of the maternal or infant vitamin metabolism, or there is a problem performing breastfeeding.
\end{abstract}

Keywords: Breast milk, colostrum, lactation, preterm birth, vitamin B12

$\mathrm{T}$ he World Health Organization recommends that infants should be exclusively breastfed for the first 6 months after birth [1]. Therefore, the micronutrient status of infants that are exclusively breastfed is dependent on breast milk. There are relatively few studies that have examined the extent to which the diet and nutrition of the mother affect the vitamin B12 content in breast milk and how the infant is affected. One rea- son may be concern that it could discourage breastfeeding if some micronutrients are found to be insufficient in breast milk [2]. However, in cases where the mother has poor nutrition, the micronutrient status of the infant is generally insufficient in the first 6 months $[3,4]$.

Vitamin B12 is found in biological matrices in either methylated forms or bound to adenosine as a coenzyme. Vitamin

Address for correspondence: Fatih Yesildal, MD. Department of Medical Biochemistry, Istanbul Medeniyet University Goztepe Teaching and Research Hospital, Istanbul, Turkey

Phone: +90 2165709023 E-mail: fyesildal@yahoo.com ORCID: 0000-0002-8738-5964

Submitted Date: January 15, 2020 Accepted Date: April 14, 2020 Available Online Date: April 29, 2020

${ }^{\circ}$ Copyright 2020 by International Journal of Medical Biochemistry - Available online at www.internationalbiochemistry.com

OPEN ACCESS This work is licensed under a Creative Commons Attribution-NonCommercial 4.0 International License. (1) 
B12 is an essential nutrient that plays a critical role in the reactions of folate-dependent single-carbon transport, including the conversion of homocysteine to methionine, the conversion of methylmalonyl coenzyme A to succinyl coenzyme A, and nucleic acid synthesis [5] Therefore, vitamin B12 is very important for growth and development in the fetal and infantile periods [6]

The transfer of vitamin B12 from blood to the mammary gland epithelium occurs through receptor-mediated endocytosis [7]. It is then secreted from the gland epithelium into the milk. Vitamin B12 is mostly bound to apo-haptocorrin in breast milk [8].

The vitamin B12 concentration in breast milk has been shown to be lower in mothers who are vitamin B12 deficient [9]. The liver of a newborn delivered of a normal, healthy mother contains $25-30 \mu \mathrm{g}$ of vitamin B12. However, if the mother has a low vitamin B12 level, the amount of vitamin B12 in the liver of the newborn infant decreases to $2-5 \mu \mathrm{g}$. The recommended dietary allowance (RDA) of vitamin B12 for the growth of tissues and neurological development is $0.4 \mu \mathrm{g}[7,10]$. The recommended daily intake of vitamin B12 for breastfeeding mothers is $2.8 \mu \mathrm{g}$ [11].

The aim of this study was to investigate whether the vitamin B12 content of breast milk differed according to the week of delivery (preterm, term) and how the vitamin B12 content in breast milk changed during the first 28 days of lactation and to assess the need for supplementary vitamin B12.

\section{Materials and Methods}

Infants born before the $34^{\text {th }}$ gestational week were classified as preterm, those delivered between the $34^{\text {th }}$ and $37^{\text {th }}$ weeks were grouped as late preterm, and the term delivery group consisted of infants born after the $37^{\text {th }}$ week. A total of 30 mothers were included in the study group, with 10 in each of the 3 groups of preterm, late preterm, term delivery. Milk samples were collected from these mothers on postpartum days 3,7 , and 28. The milk sample taken on the $3^{\text {rd }}$ day was defined as colostrum, the sample taken on the $7^{\text {th }}$ day was defined as transition milk, and the sample taken on the $28^{\text {th }}$ day was defined as mature milk. Thus, a total of 30 milk samples were collected for each delivery group. Multiple pregnancies and congenital malformations were excluded from the study. Each mother used a breast pump (Medela Mini Electric; Medela AG, Baar, Switzerland) between 8:00 and 9:00 in the morning to drain a single breast, and $4 \mathrm{~mL}$ of this milk was collected into tubes, thereby eliminating any error due to differences between foremilk and hindmilk. The tubes were centrifuged at $2000 \mathrm{~g}$ for 10 minutes at $+4^{\circ} \mathrm{C}$. Since the upper fat layer would interfere with the analysis, the supernatant below the fat layer was accessed with a fine-tip syringe and the samples were aliquoted for vitamin B12 analysis. A total of 90 breast milk samples were stored at $-80^{\circ} \mathrm{C}$ until analysis.

The statistical power was calculated as $86 \%$ with an alpha error of 0.05 and an effect size of 0.8 ( $G *$ Power 3.1.9.4 software; Faul, F., Erdfelder, E.). Vitamin B12 measurements were performed on a Siemens Advia Centaur XP autoanalyzer (Siemens Healthineers $\mathrm{GmbH}$, Erlangen, Germany). Analysis of variance (ANOVA) and post hoc Tukey tests were used for group comparisons. The Kruskal-Wallis test was used for the analysis of nonparametric data. $\mathrm{P}<0.05$ was considered statistically significant.

This study was approved by the Gazi University Clinical Research Ethics Committee on March 9, 2015 (Approval number: 2015/120). Informed consent was obtained from all of the patients included in this study. The study was conducted and the data were collected according to the ethical principles of the Declaration of Helsinki.

\section{Results}

Demographic features of the participants are presented in Table 1. The groups had similar characteristics in terms of maternal age at birth, mode of delivery, primiparity, and the sex of the infant. Birth weight, and the height and head circumference of the infants differed significantly between the preterm, late preterm, and term groups, as expected.

The vitamin B12 level in all of the breast milk samples ranged from 83 to $2000 \mathrm{ng} / \mathrm{L}$. The vitamin B12 values according to delivery group and the number of days of lactation are shown in Table 2. There was a significant difference in the vitamin B12 level between the 3 groups regardless of lactation days (ANOVA test; $p=0.012$ ) (Table 2). When post hoc Tukey pair-

Table 1. Demographic features of the participating mothers and infants

\begin{tabular}{lllll}
\hline & Preterm $(\mathbf{n = 1 0})$ & Late preterm $(\mathbf{n = 1 0})$ & Term (n=10) & p (ANOVA) \\
\hline Maternal age (years) & $28.7 \pm 4.6$ & $29.9 \pm 4.2$ & $29.6 \pm 4.6$ \\
Mode of delivery (CS/vaginal) & $8 / 2$ & $6 / 4$ & $7 / 3$ & 0.826 \\
Primiparity & 8 & 7 & 7 & $0.631^{*}$ \\
Sex of infant (male/female) & $7 / 3$ & $6 / 4$ & $4 / 6$ & $0.848^{*}$ \\
Weight at birth (g) & $1506.5 \pm 479$ & $2634.5 \pm 503.7$ & $3543 \pm 206.7$ \\
Length at birth (cm) & $39.7 \pm 4.5$ & $47 \pm 3.3$ & $49.9 \pm 0.9$ & $0.399^{*}$ \\
Head circumference $(\mathrm{cm})$ & $28.7 \pm 3.4$ & $33 \pm 1.8$ & $34.5 \pm 1.4$ & $<0.001$ \\
\hline
\end{tabular}

*Kruskal-Wallis test was used for nonparametric variables. ANOVA: Analysis of variance; CS: Cesarean section. 
Table 2. Vitamin B12 values according to the gestational week of delivery group and lactation days (ng/L)

\begin{tabular}{llllll}
\hline & $3^{\text {rd }}$ day & 7 $^{\text {th }}$ day & 28 $^{\text {th }}$ day & All days & p (ANOVA) \\
\hline Preterm & $947.6 \pm 572.7$ & $810.5 \pm 364.5$ & $1104.1 \pm 431.9$ & $954.1 \pm 462.8$ \\
Late preterm & $753.6 \pm 232.9$ & $799.5 \pm 315.5$ & $655.5 \pm 566.6$ & $736.2 \pm 385.1$ & 0.420 \\
Term & $707.5 \pm 240.5$ & $686.3 \pm 241.1$ & $547.1 \pm 274.4$ & $647 \pm 253.2$ & 0.763 \\
All deliveries & $804.8 \pm 387.6$ & $764.1 \pm 303.9$ & $773.2 \pm 485.7$ & $780.7 \pm 394.6$ \\
p (ANOVA) & 0.397 & 0.654 & 0.030 & 0.012 & 0.358 \\
\hline
\end{tabular}

Values are given as mean \pm SD. Significant $\mathrm{p}$ values are marked in bold. ANOVA: Analysis of variance.

wise comparisons were performed, a significant difference was found in the milk vitamin B12 level between the preterm delivery group and term delivery group $(p=0.010)$. When the preterm, late preterm, and term delivery groups were evaluated separately, there was no statistically significant difference according to lactation days.

There was no statistically significant difference between the 3 lactation days tested, regardless of the delivery group (ANOVA test; $p=0.928$ ) (Table 2). When ANOVA was performed to compare the delivery groups by lactation days, the only significant difference was found on the $28^{\text {th }}$ day (ANOVA test; $p=0.030$ ). Post hoc Tukey pairwise comparisons showed that the difference between the preterm and late preterm delivery groups, and the late preterm and term delivery groups was not significant ( $p=0.107$ and $p=0.866$, respectively); however, the vitamin B12 value in mature milk significantly differed between the preterm and term delivery groups $(p=0.032)$.

\section{Discussion}

Breast milk contains many growth factors, proteins, and micronutrients essential for the growth and development of infants $[1,2,12,13]$. Vitamin B12 is one of the most important micronutrients in breast milk. Lactation nutrients are examined in 2 groups. Group 1 nutrients (thiamine, riboflavin, vitamin B6, vitamin $B 12$, choline, retinol, vitamin $A$, vitamin $D$, selenium, and iodine) are usually carefully monitored. A decrease in Group 1 nutrients in the mother's blood leads directly to a decrease of these nutrients in breast milk. Dietary supplements of these nutrients for the mother also increase the level in breast milk and benefit the infant. The level of group 2 nutrients (folate, calcium, iron, copper, and zinc) in breast milk is not immediately affected by the amount in the blood of the mother or by nutritional intake. Unless the stores of mothers are completely depleted, the level of these nutrients in milk is mostly stable. Supplementation of mothers with Group 2 nutrients provides benefit to the mother rather than the infant [2].

Vitamin B12 is commonly found in breast milk in the form of methylcobalamin or deoxyadenosylcobalamin [5]. The composition of breast milk may vary depending on the gestational week of delivery and the number of days of postnatal lactation. Previous studies have examined how vitamin B12 content in breast milk changes during lactation [7, 14-16].
However, to the best of our knowledge, this is the first study to investigate the vitamin B12 level of breast milk for preterm and term delivery infants at postpartum 28 days.

Research has shown that the amount of vitamin B12 in breast milk varies widely. This may be due to factors such as dietary differences, maternal supplementation during pregnancy and lactation, and analytical differences [9]. In 1 study, it was suggested that there was no difference in the vitamin B12 level between foremilk and hindmilk [14]. However, in another study, it was reported that the amount of vitamin B12 in foremilk was lower than that of hindmilk [17]. Therefore, in this study, a breast pump was used on a single breast to obtain a homogenous mixture and this variability was eliminated.

The highest level of vitamin B12 is typically found in the colostrum, and it decreases gradually in transition milk and then remains stable in mature milk $[7,14]$. In this study, the level of vitamin B12 in breast milk of the term and late preterm delivery groups was similar over time, but a different pattern was observed in the preterm delivery group. Interestingly, the vitamin B12 level in mature milk samples collected on $28^{\text {th }}$ day of lactation was even higher than those of the colostrum. Another striking finding was that the vitamin B12 level was higher in the colostrum, transition milk, and mature milk samples in the preterm delivery group compared with the term delivery group (Fig. 1). These findings may be related to a hy-

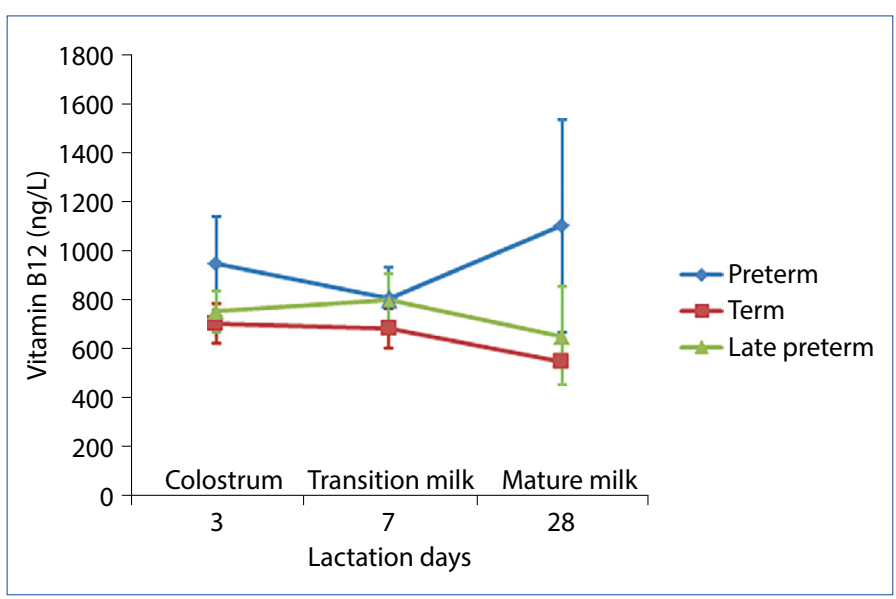

Figure 1. The variation in vitamin B12 level in the colostrum (day 3), transition milk (day 7) and mature milk (day 28) in the preterm and term delivery groups (Means are given with standard error) $\left({ }^{*} \mathrm{p}<0.05\right)$. 
pothesis that the premature infant receives more vitamin B12 support in breast milk to compensate for any possible delay in growth and development.

Vitamin B12 deficiency is a common condition in mothers and infants [18]. Vitamin B12 deficiency is usually associated with an increase in homocysteine and methylmalonic acid levels. If a mother has a vitamin B12 deficiency due to conditions such as veganism or pernicious anemia, deficiency and possible disorder can occur in the infant because the vitamin B12 level in the breast milk is insufficient. However, it may take 3-4 months for the infant to present clinical findings. The neonatal period is very sensitive to vitamin B12 deficiency [19]. This is why the use of formula or milk fortification is of great concern. Findings such as small head circumference of infants, apathy, growth and developmental retardation, and impaired motor and cognitive functions are common when there is a vitamin B12 deficiency [20]. In these cases, an intramuscular (IM) vitamin B12 injection is often preferred; however, supplementing the mother will not support the increase of vitamin B12 in the infant. Most of an IM vitamin B12 injection is retained by the mother and only a small portion passes into the milk. Therefore, it is more appropriate to provide vitamin B12 directly to the infant rather than waiting it to be transmitted in breast milk [2]. For the same reason, there is a better correlation between the vitamin B12 level in the serum of the mother and the vitamin B12 in the serum of infant rather than breast milk [21, 22].

It has been reported that the serum vitamin B12 levels of infants fed exclusively with breast milk are lower than those fed with formula [23]. However, this should not lead to discouraging opinions about breastfeeding.

The limitations of this study include the small number of participants and lack of the information about the serum level of vitamin B12 in the mothers and infants studied.

\section{Conclusion}

In conclusion, the level of vitamin B12 was highest in the colostrum and lower in the transition milk and mature milk of the term delivery group. The vitamin B12 concentration was higher in mature milk collected on the $28^{\text {th }}$ day of lactation than that of the colostrum in the preterm delivery group. In addition, it was found that the vitamin B12 level in the breast milk of the preterm delivery group was higher on the $3^{\text {rd }}$, $7^{\text {th }}$, and $28^{\text {th }}$ days of lactation compared to the term delivery group. Since vitamin B12 deficiency directly affects both the mother and infant, both should be given vitamin B12 supplementation. However, infants who receive adequate amounts of vitamin B12 in breast milk do not need to be supplemented. Especially in preterm delivery infants, unnecessary extra vitamin B12 may be given to the infant out of fear of growth and developmental retardation. According to the data obtained in this study, unless the mother lacks vitamin B12 or there is a known disorder in breastfeeding; vitamin B12 supplementation is not required even in the preterm delivery group. The serum vitamin B12 level of infants is not adjusted solely based on milk vitamin B12 content. The liver plays an important role in the storage of vitamin B12 in both the fetal and infantile periods. One should also consider the storage of vitamin B12 in the infant liver to evaluate the potential need for vitamin B12. In order to reveal the mechanisms related to these data more clearly, comprehensive studies are needed with more participants and in which breast milk vitamin B12 levels as well as serum vitamin B12 levels of both mothers and infants are evaluated during lactation.

\section{Conflict of interest: None declared.}

Ethics Committee Approval: Gazi University Clinical Research Ethics Committee 09.03.2015 with decision number 120.

Financial Disclosure: None declared.

Peer-review: Externally peer-reviewed.

Authorship contributions: Concept - F.Y., T.O., E.K., H.O.; Design - F.Y., T.O., E.K., H.O.; Supervision - F.Y., T.O., E.K., H.O.; Funding F.Y., T.O., E.K., H.O.; Materials - F.Y., T.O., E.K., H.O.; Data collection \&/or processing - F.Y., H.O.; Analysis and/or interpretation - F.Y.; Literature search - F.Y.; Writing - F.Y., T.O., E.K., H.O.; Critical review - F.Y., T.O., E.K., H.O.

\section{References}

1. Kramer MS, Kakuma R. Optimal duration of exclusive breastfeeding. Cochrane Database Syst Rev 2012;(8):CD003517.

2. Allen LH. B vitamins in breast milk: relative importance of maternal status and intake, and effects on infant status and function. Adv Nutr 2012;3(3):362-9. [CrossRef]

3. Allen $L$, Shrimpton R. The International Research on Infant Supplementation study: implications for programs and further research. J Nutr 2005;135(3):666S-9S. [CrossRef]

4. Lartey A, Manu A, Brown KH, Dewey KG. Predictors of micronutrient status among six- to twelve-month-old breast-fed Ghanaian infants. J Nutr 2000;130(2):199-207. [CrossRef]

5. Allen LH, Hampel D. Water-Soluble Vitamins in Human Milk Factors Affecting Their Concentration and Their Physiological Significance. Nestle Nutr Inst Workshop Ser 2019;90:69-81.

6. Pepper MR, Black MM. B12 in fetal development. Semin Cell Dev Biol 2011;22:619-23. [CrossRef]

7. Dror DK, Allen LH. Vitamin B-12 in Human Milk: A Systematic Review. Adv Nutr. 2018;9(suppl_1):358S-66S. [CrossRef]

8. Sandberg DP, Begley JA, Hall CA. The content, binding, and forms of vitamin B12 in milk. Am J Clin Nutr 1981;34(9):171724. [CrossRef]

9. Deegan $\mathrm{KL}$, Jones $\mathrm{KM}$, Zuleta $\mathrm{C}$, Ramirez-Zea $\mathrm{M}$, Lildballe $\mathrm{DL}$, Nexo $\mathrm{E}$, et al. Breast milk vitamin B-12 concentrations in Guatemalan women are correlated with maternal but not infant vitamin B-12 status at 12 months postpartum. J Nutr 2012;142(1):112-6. [CrossRef]

10. Institute of Medicine (US) Standing Committee on the Scientific Evaluation of Dietary Reference Intakes and its Panel on Folate, Other B Vitamins, and Choline. Dietary Reference Intakes for Thiamin, Riboflavin, Niacin, Vitamin B6, Folate, Vi- 
tamin B12, Pantothenic Acid, Biotin, and Choline. Washington (DC): National Academies Press (US); 1998.

11. Doets EL, In 't Veld PH, Szczecińska A, Dhonukshe-Rutten RA, Cavelaars $A E$, van 't Veer $P$, et al. Systematic review on daily vitamin B12 losses and bioavailability for deriving recommendations on vitamin B12 intake with the factorial approach. Ann Nutr Metab 2013;62(4):311-22. [CrossRef]

12. Hirai $C$, Ichiba $H$, Saito $M$, Shintaku H, Yamano T, Kusuda S. Trophic effect of multiple growth factors in amniotic fluid or human milk on cultured human fetal small intestinal cells. J Pediatr Gastroenterol Nutr 2002;34(5):524-8. [CrossRef]

13. Yesildal F, Koc E, Tas A, Ozgurtas T. Angiopoietins in Human Breast Milk. Breastfeed Med 2016;11:366-9. [CrossRef]

14. Hampel D, Shahab-Ferdows S, Islam MM, Peerson JM, Allen LH. Vitamin Concentrations in Human Milk Vary with Time within Feed, Circadian Rhythm, and Single-Dose Supplementation. J Nutr 2017;147(4):603-11. [CrossRef]

15. Trugo NM, Donangelo CM, Koury JC, Silva MI, Freitas LA. Concentration and distribution pattern of selected micronutrients in preterm and term milk from urban Brazilian mothers during early lactation. Eur J Clin Nutr 1988;42(6):497-507.

16. Bae S, West AA, Yan J, Jiang X, Perry CA, Malysheva $O$, et al. Vitamin B-12 Status Differs among Pregnant, Lactating, and Control Women with Equivalent Nutrient Intakes. J Nutr 2015;145(7):1507-14. [CrossRef]

17. Greibe E, Lildballe DL, Streym S, Vestergaard P, Rejnmark L, Mosekilde $L$, et al. Cobalamin and haptocorrin in human milk and cobalamin-related variables in mother and child: a 9-mo longitudinal study. Am J Clin Nutr 2013;98(2):389-95. [CrossRef]

18. McLean E, de Benoist B, Allen LH. Review of the magnitude of folate and vitamin B12 deficiencies worldwide. Food Nutr Bull 2008;29(2 Suppl):S38-S51. [CrossRef]

19. Molloy AM, Kirke PN, Brody LC, Scott JM, Mills JL. Effects of folate and vitamin B12 deficiencies during pregnancy on fetal, infant, and child development. Food Nutr Bull 2008;29(2 Suppl):S101-S15. [CrossRef]

20. Dror DK, Allen LH. Effect of vitamin B12 deficiency on neurodevelopment in infants: current knowledge and possible mechanisms. Nutr Rev 2008;66(5):250-5. [CrossRef]

21. W Widen EM, Bentley ME, Kayira D, Chasela CS, Daza EJ, Kacheche ZK, Tegha G, et al. Changes in soluble transferrin receptor and hemoglobin concentrations in Malawian mothers are associated with those values in their exclusively breastfed, HIV-exposed infants. J Nutr 2014;144(3):367-74. [CrossRef]

22. Chebaya P, Karakochuk CD, March KM, Chen NN, Stamm RA, Kroeun $\mathrm{H}$, et al. Correlations between Maternal, Breast Milk, and Infant Vitamin B12 Concentrations among Mother-Infant Dyads in Vancouver, Canada and Prey Veng, Cambodia: An Exploratory Analysis. Nutrients 2017;9(3):270. [CrossRef]

23. Karademir F, Suleymanoglu S, Ersen A, Aydinoz S, Gultepe $M$, Meral $C$, et al. Vitamin B12, folate, homocysteine and urinary methylmalonic acid levels in infants. J Int Med Res 2007;35(3):384-8. [CrossRef] 